\title{
Effects of False Tilt Cues on the Training of Manual Roll Control Skills
}

\author{
Peter M.T. Zaal* \\ San Jose State University \\ NASA Ames Research Center \\ Moffett Field, $C A$
}

\author{
Alexandru Popovici ${ }^{\dagger}$ \\ San Jose State University \\ NASA Ames Research Center \\ Moffett Field, CA \\ and \\ Delft University of Technology \\ Delft, Netherlands
}

\author{
Melinda A. Zavala ${ }^{\ddagger}$ \\ San Jose State University \\ NASA Ames Research Center \\ Moffett Field, $C A$
}

This paper describes a transfer-of-training study performed in the NASA Ames Vertical Motion Simulator. The purpose of the study was to investigate the effect of false tilt cues on training and transfer of training of manual roll control skills. Of specific interest were the skills needed to control unstable roll dynamics of a mid-size transport aircraft close to the stall point. Nineteen general aviation pilots trained on a roll control task with one of three motion conditions: no motion, roll motion only, or reduced coordinated roll motion. All pilots transferred to full coordinated roll motion in the transfer session. A novel multimodal pilot model identification technique was successfully applied to characterize how pilots' use of visual and motion cues changed over the course of training and after transfer. Pilots who trained with uncoordinated roll motion had significantly higher performance during training and after transfer, even though they experienced the false tilt cues. Furthermore, pilot control behavior significantly changed during the two sessions, as indicated by increasing visual and motion gains, and decreasing lead time constants. Pilots training without motion showed higher learning rates after transfer to the full coordinated roll motion case.

\section{Nomenclature}

$\begin{array}{llll}A_{d} & \text { disturbance amplitude, deg } & N_{d} & \text { number of disturbance sine waves } \\ A_{t} & \text { target amplitude, deg } & N_{t} & \text { number of target sine waves } \\ e & \text { error signal, deg } & n & \text { pilot remnant, deg } \\ f_{d} & \text { disturbance forcing function, deg } & i_{r} & \text { run number } \\ f_{t} & \text { target forcing function, deg } & s & \text { Laplace operator } \\ g & \text { standard gravity, ft s }{ }^{-2} & T_{L} & \text { lead time constant, s } \\ H_{c} & \text { aircraft dynamics response } & T_{m} & \text { measurement time, s } \\ H_{m} & \text { pilot motion response } & u & \text { pilot control input, deg } \\ H_{o l, d} & \text { disturbance open-loop response } & y_{s} & \text { simulator lateral displacement command, ft } \\ H_{o l, t} & \text { target open-loop response } & & \\ H_{v} & \text { pilot visual response } & & \\ H_{V M S} & \text { VMS motion logic dynamics } & S y m b o l s & \\ K_{m} & \text { pilot motion gain, - } & & \\ K_{v} & \text { pilot visual gain, - } & \zeta_{n m} & \text { neuromuscular damping, }- \\ K_{r} & \text { simulator roll motion gain, }- & \zeta_{r} & \text { roll washout filter damping ratio, }- \\ K_{y} & \text { simulator lateral motion gain, - } & \lambda & \text { rate of exponential decay or growth, }- \\ N_{0} & \text { initial value level } & \tau_{m} & \text { motion delay, s } \\ N_{\infty} & \text { asymptotic level } & \tau_{v} & \text { visual delay, s }\end{array}$

\footnotetext{
${ }^{*}$ Senior Research Engineer, Human Systems Integration Division, NASA Ames Research Center, Moffett Field, CA, 94035; peter.m.t.zaal@nasa.gov. Member.

${ }^{\dagger}$ Research Scholar, Human Systems Integration Division, NASA Ames Research Center, Moffett Field, CA, 94035; a.popovici@student.tudelft.nl.

${ }^{\ddagger}$ Research Associate, Human Systems Integration Division, NASA Ames Research Center, Moffett Field, CA, 94035; melinda.a.zavala@nasa.gov.
} 


\begin{tabular}{|c|c|c|c|}
\hline$\phi$ & roll angle, deg & $\omega_{c, t}$ & target crossover frequency, $\mathrm{rad} \mathrm{s}^{-1}$ \\
\hline$\phi_{s}$ & simulator roll command, deg & $\omega_{d}$ & disturbance sinusoid frequency, $\operatorname{rad~s}^{-1}$ \\
\hline$\varphi_{m, d}$ & target phase margin, deg & $\omega_{m}$ & measurement time base frequency, $\operatorname{rad~s}^{-1}$ \\
\hline$\varphi_{m, t}$ & disturbance phase margin, deg & $\omega_{n m}$ & neuromuscular peak frequency, $\operatorname{rad~s}^{-1}$ \\
\hline$\omega$ & frequency, $\operatorname{rad~s}^{-1}$ & $\omega_{r}$ & roll washout filter break frequency, $\mathrm{rad} \mathrm{s}^{-1}$ \\
\hline$\omega_{c, d}$ & disturbance crossover frequency, $\operatorname{rad~s}^{-1}$ & $\omega_{t}$ & target sinusoid frequency, $\operatorname{rad~s}^{-1}$ \\
\hline
\end{tabular}

\section{Introduction}

Airline pilots will soon be required to perform full stall recovery training in simulators. However, flight simulators currently used for pilot training do not represent aircraft behavior in upset situations that take the aircraft out of its normal flight envelope. Post-stall aircraft models need to be implemented to correctly simulate the aircraft response after the stall point. In addition, motion cues need to adequately represent this response to ensure the skills learned in simulator training are directly usable in real flight. ${ }^{1}$ This paper investigates how different motion configurations affect training and transfer of training of the manual control skills required to control unstable roll dynamics of a mediumsize transport aircraft near the stall point. We specifically focus on the false tilt cues that are often encountered in current hexapod training simulators.

Previous research showed that simulator motion significantly affects multimodal pilot control behavior and performance. $^{2-5}$ Motion feedback provides more rapid information than visual feedback on the rate of change of the controlled aircraft state, allowing a human operator to increase disturbance-rejection performance. Little research has been performed on the effects of motion on training and transfer of training of manual control skills. Levison used pilot modeling techniques to characterize how pilot control behavior develops over the course of training and after transfer. ${ }^{6}$ A novel multimodal pilot model identification technique was successfully applied by Pool et al. to characterize changes in multimodal pilot control behavior during training. ${ }^{7}$ This approach will also be used in the current study.

The paper is structured as follows. First, a brief overview of roll motion cueing considerations is given. Then, the paper describes the experimental design, which used the world's largest flight simulator. After presentation of the results, a discussion follows.

\section{Roll Motion Cueing}

In flight simulators, gains and washout filters attenuate the aircraft model rotational and translational accelerations to keep the motion platform within its displacement, velocity and acceleration limits. The tuning of the gains and filter break frequencies always requires a tradeoff between different degrees of freedom. This is even more so for hexapod motion platforms for which the same six actuators are required to move the platform in each degree of freedom. In addition, the rotational and translational accelerations in certain degrees of freedom, such as roll and lateral, are coupled and need to be treated together in the motion tuning process. First, a certain amount of lateral translational motion must accompany a rolling motion to translate the center of rotation properly. Second, roll-tilt coordination is often used to simulate sustained lateral accelerations. Finally, for coordinated roll motion, lateral platform motion provides an acceleration to counteract the false tilt cue that would result from only rolling the cockpit. ${ }^{8,9}$

During a coordinated turn in an aircraft, the centrifugal acceleration will cancel out the tilt component of gravity in the body reference frame, keeping the apparent gravity vector $g^{\prime}$ parallel to the pilot's spine. In order to accurately reproduce this in a simulator, substantial lateral travel is required. However, due to the limited motion space of hexapod motion platforms, this compensatory lateral acceleration $[\mathrm{g} \tan (\phi)]$ can only be provided for a very limited amount of time (Fig. 1b). In the case where no lateral accelerations are provided, as depicted in Fig. 1c, the pilot perceives the horizontal component of the gravity vector and feels a false tilt cue $[g \sin (\phi)]$.

To reduce the amount of lateral displacement used to eliminate the false tilt cues, different tradeoffs can be made between motion tuning parameters in the roll and lateral degrees of freedom. First, the roll angle of the motion platform can be reduced relative to the model (and thus the visual scene). This requires less lateral travel, since the magnitude of the tilt cue is reduced. Second, the amount of lateral translational motion required for coordination can be reduced, allowing for some false cues to occur. Previous research has investigated the tradeoff between these two options and looked at the effects on pilot performance and subjective ratings. ${ }^{9}$ Another study looked at the effects of various degrees of lateral acceleration washout in a roll control task on pilot control behavior and performance. ${ }^{8}$ In both studies, the effects of false tilt cues on pilot performance were limited, and in the later study the effects on manual 
(a) No motion.

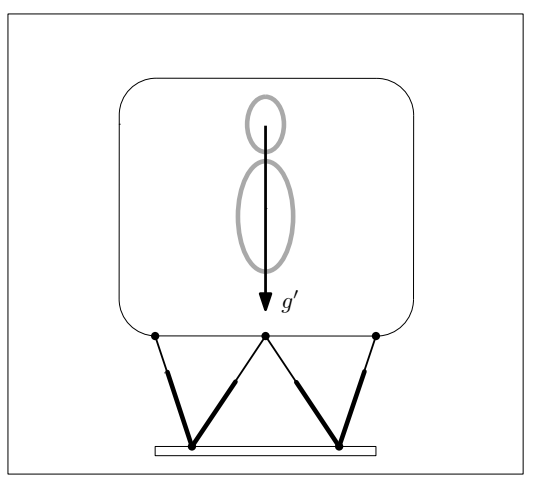

(b) Coordinated roll.

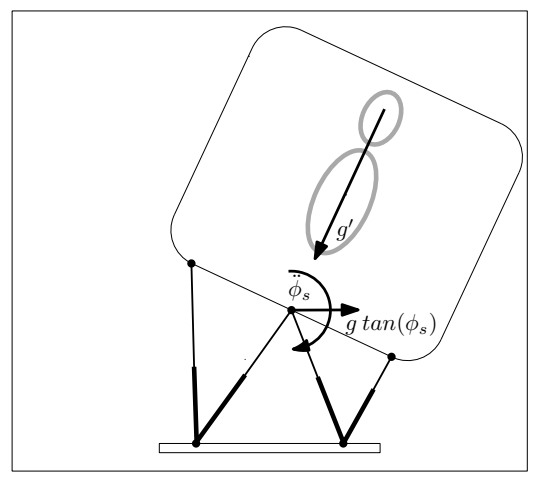

(c) Uncoordinated roll.

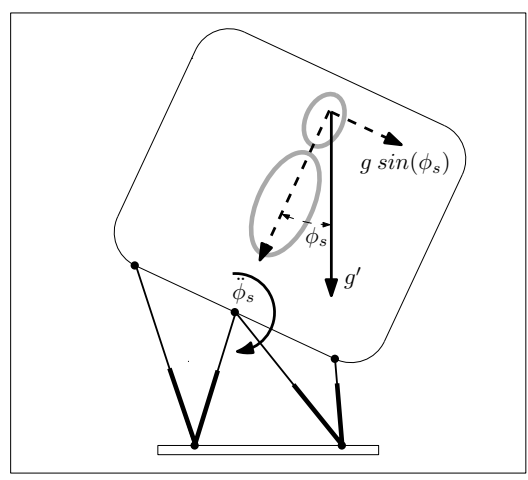

Figure 1. Apparent gravity orientation during a coordinated and uncoordinated roll in a flight simulator.

control behavior were also limited. However, significant differences were found between pilots' subjective ratings in both studies, with pilots consistently noticing the false tilt cues when the peak spurious lateral specific force was above $0.1 \mathrm{~g}$.

Although previous studies did not find many significant differences in pilot performance and control behavior, the effects of false tilt cues on pilot training and transfer of training have never been investigated. This is the purpose of the current study for which an experiment was performed on the Vertical Motion Simulator (VMS) at NASA Ames Research Center. We specifically analyzed the control of unstable roll dynamics of a transport aircraft near the stall point. For such unstable dynamics, motion has been shown to significantly affect manual control behavior and performance. In order to analyze the effect on transfer of training of manual roll control skills, pilots first trained with one of the three motion configurations visualized in Fig. 1. All these motion configurations were tuned in such a way that they could be performed on a medium-size hexapod motion base with 60-inch actuators. For the coordinated roll motion condition, this meant that roll accelerations were significantly attenuated to allow for full compensation of the lateral tilt cues. The no-motion configuration was performed as a baseline condition. After the training session, each pilot performed a transfer session, which utilized the entire lateral track of the VMS for full coordinated roll motion. More details on the motion configurations are given in Section III.

\section{Experimental Design}

\section{III.A. Control Task}

Fig. 2 depicts the roll control task performed by pilots in the VMS. The pilots' task was to actively minimize the roll error, $e$, presented on a compensatory display, which resembled a basic Primary Flight Display (PFD). In addition to the visual stimuli presented on the display, roll stimuli were provided by the simulator motion logic with dynamics $H_{V M S}$. The level of motion feedback pilots received varied according to the motion configurations presented in Fig. 1. Pilots acted on the controlled roll dynamics, $H_{c}$, by making control inputs, $u$, with a sidestick.

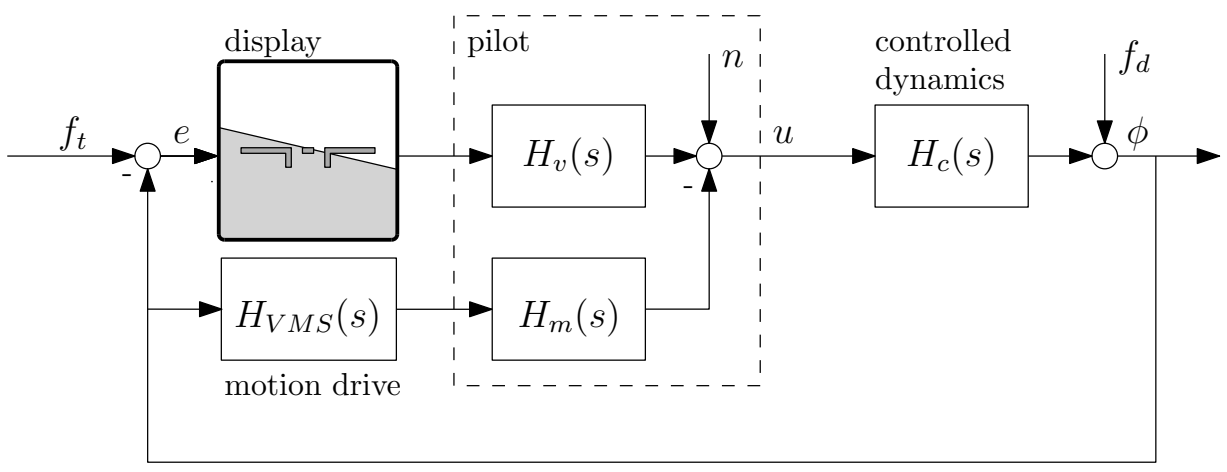

Figure 2. Control Task. 
In the control task of Fig. 2, a human operator can be modeled by linear response functions. ${ }^{10}$ A remnant signal, $n$, accounts for measurement noise and nonlinearities not captured by the response functions. In order to separate pilots' visual and motion responses, $H_{v}$ and $H_{m}$, two independent sum-of-sines forcing functions were used in the control task. ${ }^{2}$ A disturbance forcing function, $f_{d}$, was added to the controlled element output, feeding directly into the display and VMS motion system. A target forcing function, $f_{t}$, feeds into the display only through the error, $e$, the difference between $f_{t}$ and the disturbed roll attitude, $\phi$. The disturbance forcing function had a power four times greater than the target forcing function, making the control task primarily a disturbance-rejection task.

\section{III.A.1. Controlled Aircraft Dynamics}

Pilots controlled the dynamics of a mid-size twin-engine commercial transport aircraft with a gross weight of 185,800 lbs. The aircraft model was linearized close to the stall point, at an altitude of $41,000 \mathrm{ft}$ and an airspeed of $150 \mathrm{kts}$. At this flight condition, the roll dynamics of the aircraft are unstable, and defined by the following transfer function:

$$
H_{c}(s)=0.78208 \frac{\left(s^{2}+0.2175 s+0.5861\right)}{(s+0.7599)(s-0.02004)\left(s^{2}+0.1133+0.6375\right)}
$$

where $s$ represents the Laplace operator. Figure 3 depicts the frequency response of this transfer function. At low frequencies, $<0.75 \mathrm{rad} / \mathrm{s}$, the dynamics approximate a single integrator $\left(\frac{1}{s}\right)$, whereas at frequencies above $1.0 \mathrm{rad} / \mathrm{s}$ they approximate a double integrator $\left(\frac{1}{s^{2}}\right)$.

\section{III.A.2. Forcing Functions}

The target and disturbance forcing functions are sum-of-sines signals defined by:

$$
f_{d, t}(t)=\sum_{k=1}^{N_{d, t}} A_{d, t}(k) \sin \left[\omega_{d, t}(k) t+\phi_{d, t}(k)\right]
$$

where $A(k), \omega(k)$, and $\phi(k)$, respectively, indicate the amplitude, frequency, and phase of the $k^{t h}$ sine in $f_{d}$ or $f_{t}$. $N_{d, t}$ represents the number of sine waves. Subscripts $d$ and $t$ are the disturbance and target forcing function, respectively. Both $f_{d}$ and $f_{t}$ consist of ten individual sinusoids with a different amplitude, frequency, and phase. A summary of all forcing function properties can be found in Table 1.

The frequencies of the sinusoids, $\omega_{d}(k)$ and $\omega_{t}(k)$, were all integer multiples of the measurement time base frequency, $\omega_{m}=2 \pi / T_{m}=0.0767 \mathrm{rad} / \mathrm{s}$, where $T_{m}=81.92 \mathrm{~s}$ is the measurement time. The selected integer multiples were used in a number of earlier experiments and ensured that the ten sinusoid frequencies in each signal covered the frequency range of human control at regular intervals on a logarithmic scale.

A second-order low-pass filter was used to determine the amplitudes of the individual sines for both the target and the disturbance forcing functions. ${ }^{3}$ This second-order filter reduces the magnitude of the amplitudes at the higher frequencies, yielding a tracking task that is not overly difficult. The amplitude distributions $A_{d}(k)$ and $A_{t}(k)$ were scaled to attain variances for $f_{d}$ and $f_{t}$ of $2.0 \mathrm{deg}^{2}$ and $0.5 \mathrm{deg}^{2}$, respectively; meaning the target had only $25 \%$ of the
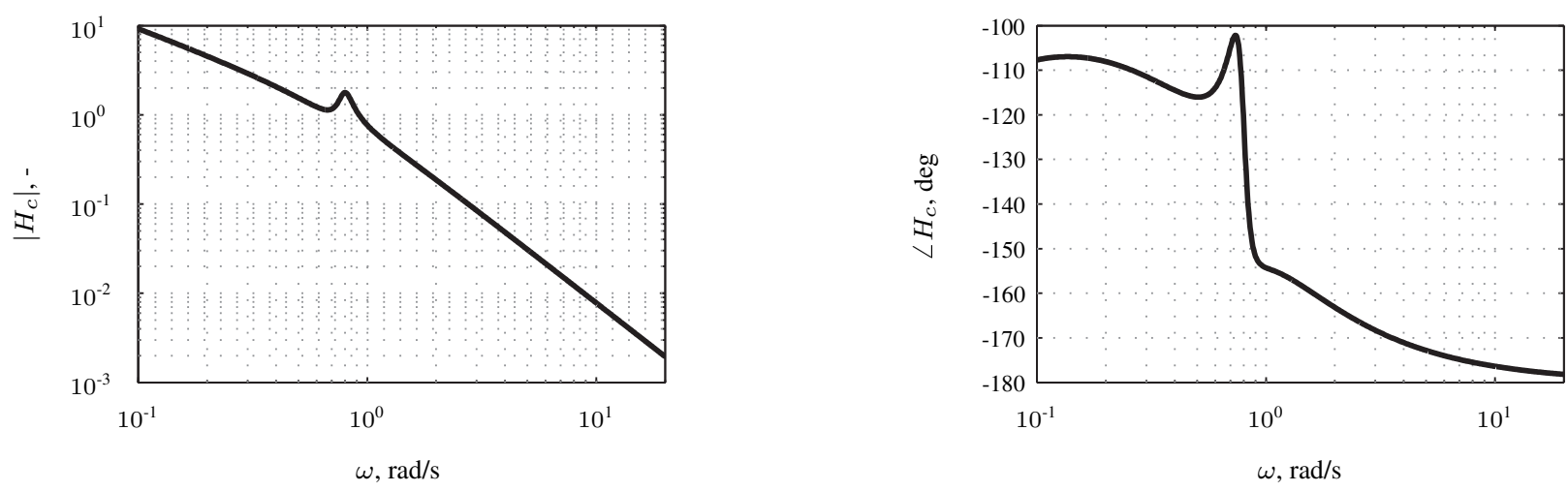

Figure 3. Frequency response dynamics. 
Table 1. Forcing function properties.

\begin{tabular}{rcccrccc}
\hline \hline \multicolumn{4}{c}{ target, $f_{t}$} \\
\hline$n_{t},-$ & $\omega_{t}$, rad $s^{-1}$ & $A_{t}, \mathrm{deg}$ & $\phi_{t}, \mathrm{rad}$ & $n_{d},-$ & $\omega_{d}$, rad $s^{-1}$ & $A_{d}, \mathrm{deg}$ & $\phi_{d}, \mathrm{rad}$ \\
\hline 6 & 0.460194 & 0.7809 & -1.4796 & 5 & 0.383495 & 1.5021 & -0.7257 \\
13 & 0.997088 & 0.5461 & -0.0745 & 11 & 0.843689 & 1.1355 & -0.9253 \\
27 & 2.070874 & 0.2464 & 0.7006 & 23 & 1.764078 & 0.5656 & 0.6432 \\
41 & 3.144661 & 0.1327 & -1.9563 & 37 & 2.837864 & 0.2881 & 2.7264 \\
53 & 4.065049 & 0.0891 & -2.8131 & 51 & 3.911651 & 0.1753 & -0.1488 \\
73 & 5.59903 & 0.0552 & 2.1026 & 71 & 5.445632 & 0.1065 & 1.8202 \\
103 & 7.900001 & 0.0351 & -2.6178 & 101 & 7.746603 & 0.0666 & -2.0536 \\
139 & 10.66117 & 0.0256 & 2.255 & 137 & 10.50777 & 0.0482 & 1.9364 \\
174 & 13.34563 & 0.0214 & -0.6739 & 171 & 13.11554 & 0.0402 & 1.0396 \\
229 & 17.56408 & 0.0182 & 0.1942 & 226 & 17.33398 & 0.034 & -2.6909 \\
\hline \hline
\end{tabular}

power of the disturbance. With a small enough variance for the target signal, the pilot will not be consciously aware of the visual/motion discrepancies and will operate as if there were only the disturbance signal. However, if the variance is too small, signal-to-noise problems will arise. This relative strength of the target and disturbance signals, and the amplitude variation determined by the second-order filter were successfully applied to measure pilot tracking behavior in earlier experiments. ${ }^{3,5}$

To determine the forcing function phase distributions, numerous random sets of phases were generated. Two sets of phases were chosen for the target and disturbance forcing functions that yielded signals with a Gaussian-like distribution and an average crest factor. ${ }^{11}$

\section{III.B. Apparatus}

This experiment used the VMS with the rotorcraft cab (R-CAB), see Fig. $4 .{ }^{12}$ A summary of VMS capabilities can be found in Ref. 13. Pilots were seated on a chair positioned in the center of the cab and controlled the roll attitude through a joystick located on the right side of the seat as seen in Fig. 5. Pilots had a simplified PFD in front of them that displayed the roll error (see Fig. 6). The out-the-window view consisted of a green ground plane and monotone blue sky. No other visual features were present in the out-the-window view.

The collimated out-the-window display of R-CAB consists of three cathode ray tube monitors casting a highquality image on three spherical mirrors. The three windows span a field of view from $+78^{\circ}$ to $-77^{\circ}$ in azimuth and $+12^{\circ}$ to $-17^{\circ}$ in elevation. The visual system equivalent time delay was $62 \mathrm{~ms}$, which is similar to the time delays of the motion system. ${ }^{13}$

\section{III.C. Experimental Conditions}

For each pilot, the experiment consisted of a training and a transfer session. The motion configuration used during the training session was the only independent variable of the experiment. Three different motion configurations were used for training: no motion, roll motion only, and coordinated roll motion (see Section II). All these motion training conditions were tuned in such a way that the motion could be executed on a medium-size hexapod motion base. Full

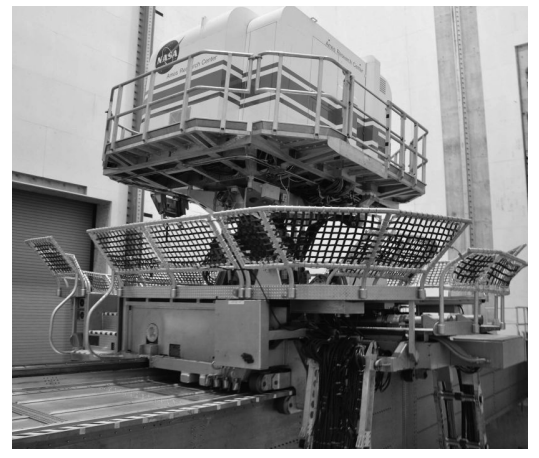

Figure 4. Vertical Motion Simulator.

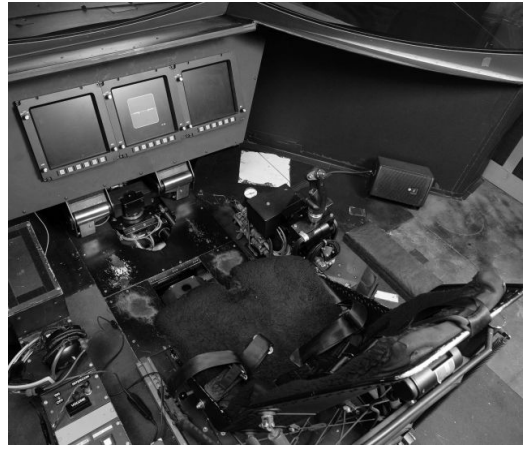

Figure 5. Cockpit setup.

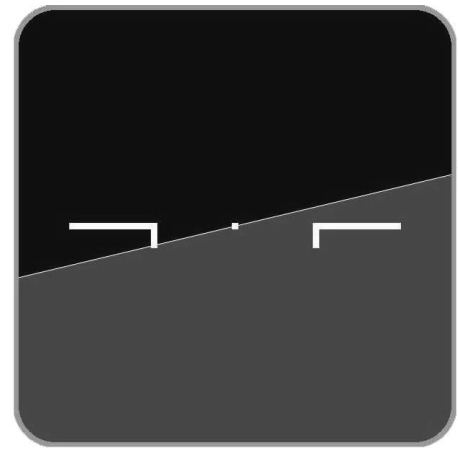

Figure 6. Primary flight display. 
coordinated roll motion was used in the transfer session after each pilot trained on one of the aforementioned training conditions. Table 2 summarizes the training motion conditions.

The motion logic used in the experiment is depicted in Fig. 7. $K_{r}$ is the roll motion gain, and $\zeta_{r}$ and $\omega_{r}$ are the damping ratio and break frequency of the roll washout filter, respectively. $K_{y}$ is a gain on the lateral accelerations. Note that Fig. 7 only provides an overview of the motion logic most relevant to this experiment. The full motion logic contained algorithms to transform linear and rotational accelerations from the body to inertial reference frame and to translate the center of rotation. The center of rotation was located $3.75 \mathrm{ft}$ below the pilot eye point in all motion configurations. Table 2 provides the values of the motion logic parameters for each motion condition. Note that the roll washout was also used for the roll-motion-only and full-coordinated-roll-motion configurations, but with a very low break frequency.

Table 2. Training motion groups.

\begin{tabular}{cllrll}
\hline \hline Group & Label & \multicolumn{2}{c}{ Training Motion $\left(K_{r}, \zeta_{r}, \omega_{r}, K_{y}\right)$} & \multicolumn{2}{c}{ Transfer Motion $\left(K_{r}, \zeta_{r}, \omega_{r}, K_{y}\right)$} \\
\hline 1 & NM & no motion & $(0.0,-,-,-)$ & full coordinated roll motion & $(1.0,0.707,0.2,1.0)$ \\
2 & RO & roll motion only & $(1.0,0.707,0.2,0.0)$ & full coordinated roll motion & $(1.0,0.707,0.2,1.0)$ \\
3 & CR & hexapod coordinated roll motion & $(0.5,0.707,0.8,1.0)$ & full coordinated roll motion & $(1.0,0.707,0.2,1.0)$ \\
\hline \hline
\end{tabular}

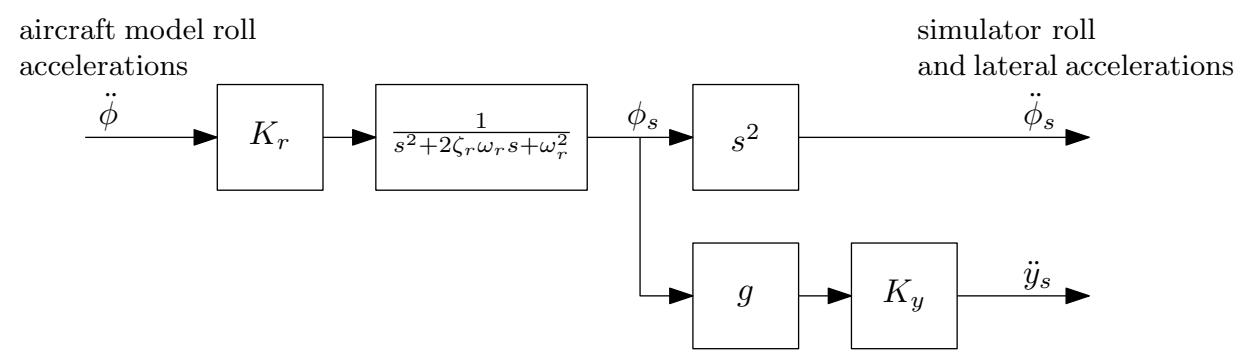

Figure 7. Simulator motion logic.

\section{III.D. Participants and Procedures}

Nineteen general aviation pilots participated. Three pilots had 1,500 or more hours of flying, with the highest number of hours at 2,000. The average number of flight hours was 534 with a standard deviation of \pm 654 . Most pilots had fixed-base simulator experience, with an average of 213 hours and standard deviation of \pm 302 . Pilots fell between the ages of 20 and 70, with the majority under 40 years with an average age of 28.8 years and a standard deviation of \pm 12.6 years.

Before the start of the experiment, each pilot received a thorough briefing informing them of the purpose of the experiment and overall procedures. In addition, the briefing explained the goal of the task and suggestions on how to compensate for the disturbances more effectively. Pilots were not given any details about the motion conditions. To motivate pilots to improve their performance, they were told the current best score and encouraged to improve on it. The pilots were also briefed on good control strategies and given a few examples on paper of good and bad control inputs. Finally, pilots were instructed to focus on the PFD (heads down) and not use the out-the-window view.

For the experiment itself, each pilot came in for two sessions spread over two days, in the morning or afternoon. For the first session, the pilot performed training for the task with one of three training conditions mentioned above. Each pilot was assigned to a motion group randomly. The next day, the pilot performed the task with the full coordinated roll motion condition. Each pilot performed 80, 90-second runs per session, in four sets of 20 with a ten-minute break between sets, for a total of 160 runs over the two sessions. During each session, the pilots were allowed to take longer breaks if they requested.

At the end of each run, the root mean square (RMS) of the roll error appeared at the bottom right of the PFD to give pilots feedback on their performance. The smaller their score, the better performance they achieved throughout that run. 


\section{III.E. Pilot Control Behavior and Performance Measures}

Several dependent measures were calculated for every pilot to evaluate how control behavior and performance changed during training and after transfer. Pilot control intensity and performance were evaluated in terms of the root mean square of the lateral control input, $R M S_{u}$, and roll error, $R M S_{e}$, respectively. These two measures were calculated for every single run.

In order to characterize pilot control behavior for each pilot, linear transfer functions are used to describe the visual and motion responses. Nonlinear behavior and measurement noise are captured by the remnant signal $n$ (Fig. 2). According to the crossover model theorem, ${ }^{10}$ a human operator adjusts his or her control behavior to the controlled dynamics such that the pilot-vehicle open-loop response approximates a single integrator near the crossover frequency. The controlled dynamics in Eq. (1) approximate a double integrator in the frequency range where the pilot-vehicle crossover frequency is expected for compensatory tracking $(1-5 \mathrm{rad} / \mathrm{s})$. This means pilots need to generate lead by using visual and, when available, motion cues.

Taking into account the need for visual lead generation, the visual response transfer function used to model manual control behavior in Fig. 2 is defined by:

$$
H_{v}(s)=K_{v}\left(T_{L} s+1\right) e^{-\tau_{v} s} \frac{\omega_{n m}^{2}}{s^{2}+2 \zeta_{n m} \omega_{n m} s+\omega_{n m}^{2}}
$$

and the motion response transfer function by:

$$
H_{m}(s)=s K_{m} e^{-\tau_{m} s} \frac{\omega_{n m}^{2}}{s^{2}+2 \zeta_{n m} \omega_{n m} s+\omega_{n m}^{2}}
$$

In this multimodal pilot model representation, pilot equalization is defined by the visual gain $K_{v}$, the visual lead time constant $T_{L}$, and the motion gain $K_{m}$. The pilot's limitations are characterized by the visual delay $\tau_{v}$, the motion delay $\tau_{m}$, and the neuromuscular dynamics with a neuromuscular frequency $\omega_{n m}$ and a neuromuscular damping $\zeta_{n m}$.

It must be noted that $H_{m}$ represents the sum of multiple separate motion feedback channels defining the use of angular accelerations detected by the semicircular canals, linear accelerations detected by the otoliths, and motion cues from the somatosensory system. This modeling approach has successfully been used in previous research. ${ }^{14}$ Furthermore, except for the VMS motion system dynamics $H_{V M S}$, all other simulator dynamics, such as latencies, are included in $H_{v}$ and $H_{m}$.

The pilot model defined in Eqs. 3 and 4 contains seven parameters $\left(K_{v}, T_{L}, \tau_{v}, K_{m}, \tau_{m}, \zeta_{n m}\right.$, and $\left.\omega_{n m}\right)$, which were estimated from experiment data using maximum likelihood estimation (MLE). Data were averaged over four measurement runs before proceeding with the parameter estimation in order to reduce the influence of the remnant on accuracy of the parameter estimates. With 80 runs, this means that control behavior was evaluated 20 times in the training and transfer sessions for each pilot. First, a genetic algorithm optimization was used to obtain rough initial estimates of the parameters. Following, a Gauss-Newton optimization algorithm obtained the set of parameters that provides the pilot model that best fit the experiment data. ${ }^{15}$ The control signal variance accounted for (VAF) was calculated as a measure of the accuracy of the pilot model in describing the measured control signal data.

In the frequency domain, pilot performance in attenuating the target and disturbance signals is determined by the crossover frequencies and phase margins of the target and disturbance open-loop dynamics, respectively. ${ }^{16}$ Using the control task diagram in Fig. 2 and the pilot response functions in Eqs. 3 and 4, the disturbance and target open-loop responses are given by:

$$
\begin{gathered}
H_{o l, d}(s)=\left[H_{v}(s)+H_{V M S}(s) H_{m}(s)\right] H_{c}(s) \\
H_{o l, t}(s)=\frac{H_{v}(s) H_{c}(s)}{1+H_{V M S}(s) H_{m}(s) H_{c}(s)}
\end{gathered}
$$

The disturbance and target crossover frequencies, $\omega_{c, d}$ and $\omega_{c, t}$, are the frequencies where the magnitude of the disturbance and target open-loop responses is 1.0. The corresponding phase margins, $\varphi_{m, d}$ and $\varphi_{m, t}$, are the phase differences from -180 degrees at these crossover frequencies.

For each pilot, an exponential decay function was fit on all the dependent measures described above to characterize each measure's change during training or transfer sessions:

$$
N\left(i_{r}\right)=\left(N_{0}-N_{\infty}\right) e^{-\lambda i_{r}}+N_{\infty}
$$


where $N_{0}$ is the initial value of the measure at the start of the session, $N_{\infty}$ is the asymptotic value at the end of a session, $\lambda$ is the rate of exponential decay or growth, and $i_{r}$ is the run number.

\section{Results}

This section provides the results of 14 of the 19 pilots who participated in the experiment. After a thorough analysis, five pilots were eliminated from the data analysis for the following reasons: one pilot did not finish the experiment, two performed below standards, and two produced control inputs that did not allow for accurate pilot model parameter estimates. This left four pilots in groups NM and CR, and six pilots in group RO.

As described in Section III.E, an exponential decay function was fit to each of the dependent measures for both the training and transfer sessions of the experiment. An example of a curve fit applied to the performance of one pilot in the training and transfer sessions is depicted in Fig. 8. This curve fitting method is particularly useful since it provides a measure of the initial performance level $N_{0}$, the theoretical asymptotic performance reached at the end of the session $N_{\infty}$, and the learning rate $\lambda$. These parameters can be used to analyze the differences in training and transfer of training from the different training motion configurations. An analysis of variance (ANOVA) was performed on each dependent measure to determine whether differences found between motion groups were statistically significant. A summary of the ANOVA results can be found in the appendix.

(a) Training.

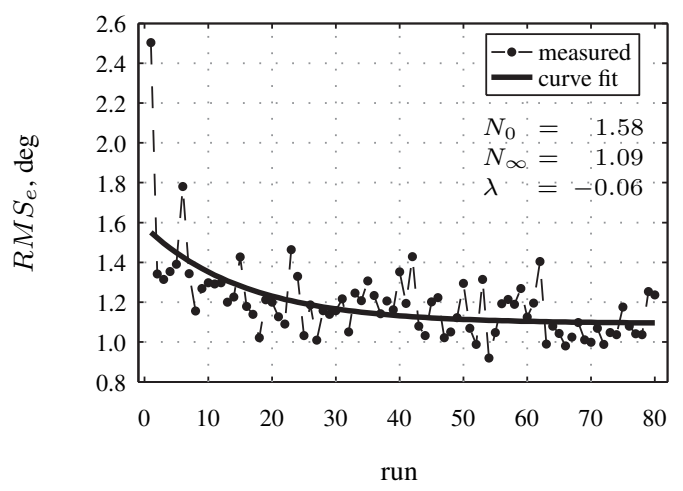

(b) Transfer.

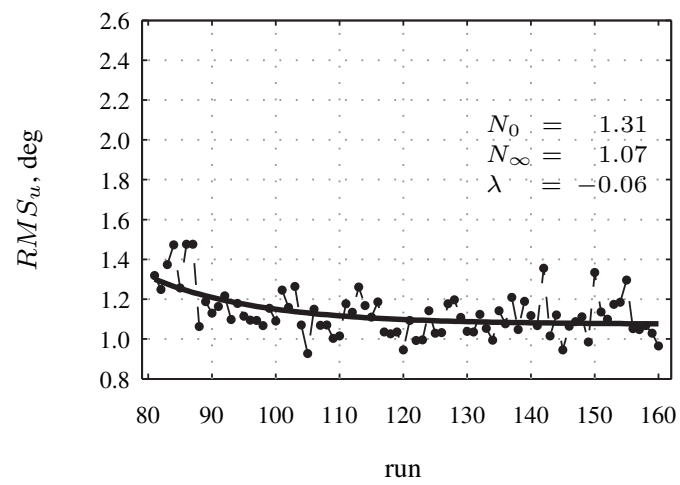

Figure 8. Example curve fit.

\section{IV.A. Performance and Control Activity}

Pilot performance is defined as the RMS of the roll error presented on the PFD. The lower the value of $R M S_{e}$, the better the performance. Fig. 9a presents the evolution of performance during training and transfer. The curves depicted in Fig. 9a are constructed using the curve fitting parameters averaged over all pilots of a motion group. The means and 95\% confidence intervals of the initial and asymptotic performance levels for each group are depicted at the start and end of each curve. The confidence intervals are corrected for small sample size.

In the training phase, the roll-only motion condition yields the best performance. Furthermore, the error bars are the smallest out of all conditions, indicating that in the roll-only condition, pilot performance varies less. The variance in pilot performance appears to decrease overall in the transfer condition compared to training. Performance in the coordinated-roll-training-motion condition (CR) is very similar to performance in the no-motion condition (NM). Note that the order of performance levels between motion groups seems to be maintained in the transfer session. In addition, Fig. 9a suggests that pilots in the NM and CR motion groups took longer to converge to asymptotic performance in the transfer session.

Fig. 9b depicts the RMS of pilots' control input. Pilots in the no-motion group seem to control with the lowest intensity during training, and have the smallest variance between pilots. In the transfer session, the RO motion group has the smallest variance between pilots. This suggests that all pilots who trained with roll motion only control with similar intensity in the fully coordinated roll motion condition. Pilots who trained with no motion or reduced coordinated roll motion showed much larger variations in control intensity in the transfer session. 
(a) Roll error RMS.

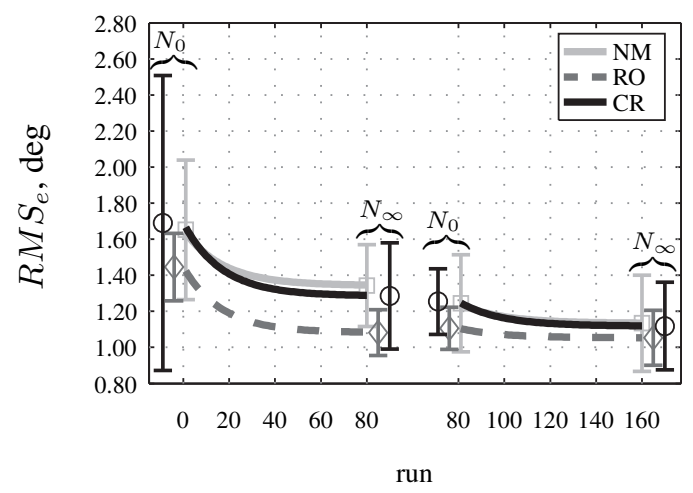

(b) Stick deflection RMS.

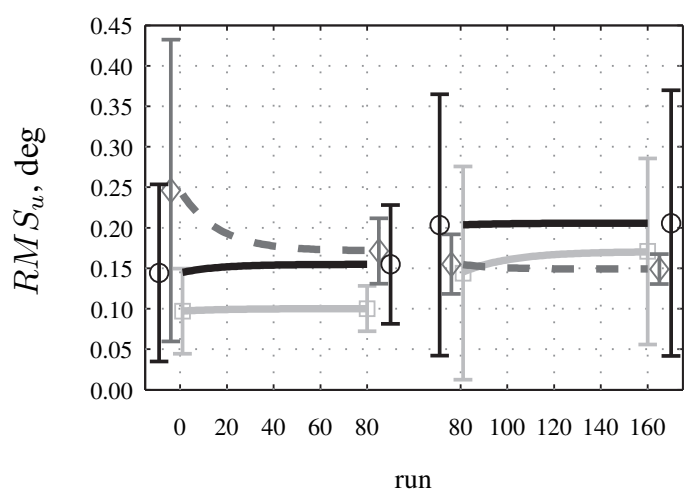

Figure 9. Pilot performance and control activity.

\section{IV.B. Variance Accounted For}

The VAF indicates how much of the variance present in the measured signal can be accounted for by the linear pilot model described in Section III.E. Fig. 10 depicts the evolution of the VAF for all three training motion groups in the training and transfer sessions. Again, the means and $95 \%$ confidence intervals of the initial and asymptotic values are depicted at both ends of the exponential curves. The figure suggests that the VAF at the start of the training session is similar for all three motion groups. For the RO and CR motion groups, the VAF level has an increasing trend during the training session, which suggests that the pilots adopt a more linear and constant control strategy while training. At the end of the training session, the highest VAF is achieved for the CR motion group, whereas the VAF for the NM group is the lowest. This result indicates that pilots adopt a more linear control strategy when training with reduced coordinated roll motion. The relative order of the VAF is maintained in the transfer session, where full coordinated roll motion was present. Fig. 10 also shows that the variance between pilots becomes smaller at the end of training compared to the variance at the beginning.

\section{IV.C. Pilot Model Parameters.}

The estimated model parameters from the pilot model presented in Section III.E are depicted in Fig. 11, which shows the evolution of all the parameters in the training and transfer sessions. The curves are obtained using the mean curve parameters for each motion group. The error bars indicate the $95 \%$ confidence intervals, corrected for small sample size, for the initial and asymptotic levels of each curve.

The visual gain of the pilot is depicted in Fig. 11a. From this figure one can see that the group who trained without motion has the lowest visual gain during training. When motion is present during training, the visual gain is higher, suggesting that pilots are more confident to act on visual information when performing the task.

Fig. 11b depicts the lead time constant evolution for each of the motion groups. During training, the group learning without motion yields the largest value for this parameter, indicating that this group generates the most lead information from the visual stimuli. This is expected, since the visual stimuli are the only stimuli pilots can use to obtain lead information. While the roll-only motion condition had the largest miscues, the group training with this motion had the lowest lead time constant, indicating that pilots generate the least visual lead. The value of the lead time constant for the coordinated-roll training group lies between the values for the two other groups. Furthermore, the relative order of the lead time constant values between the different groups in the training session is the opposite of what was observed for the visual gain. At the end of the transfer session, all three levels of the lead time constant seem to converge to the same value. However, the NM group starts with the highest lead time constant in the transfer session and has the highest learning rate. 
(a) Visual gain.

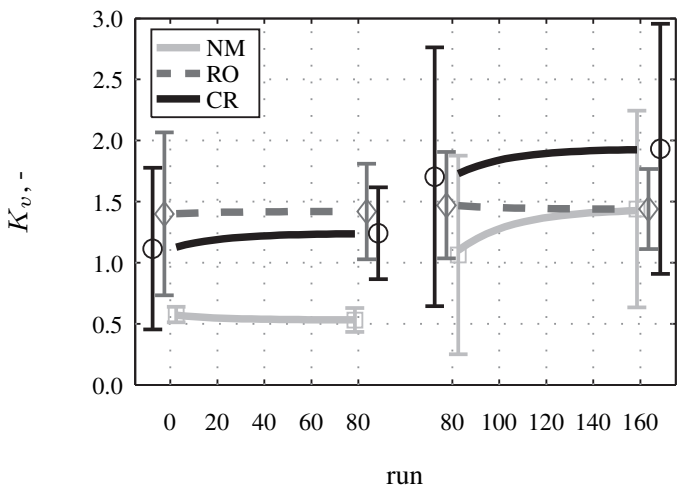

(b) Lead time constant.

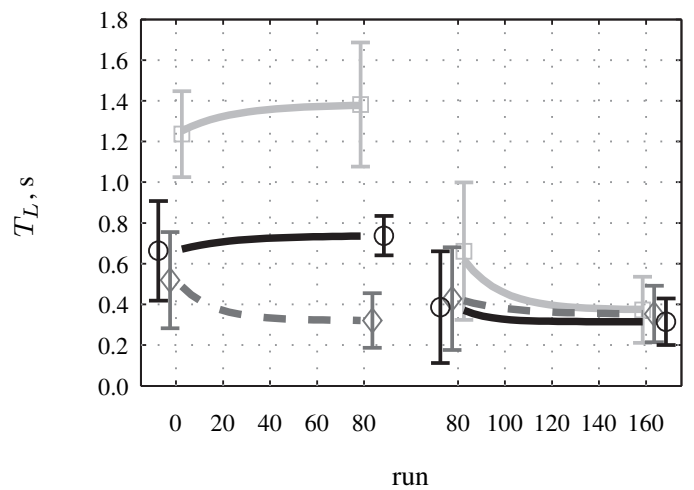

(c) Visual delay.

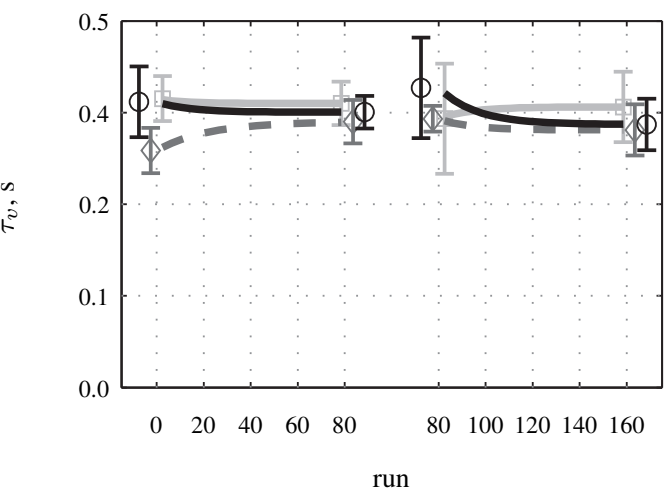

(d) Motion gain.

(e) Motion delay.

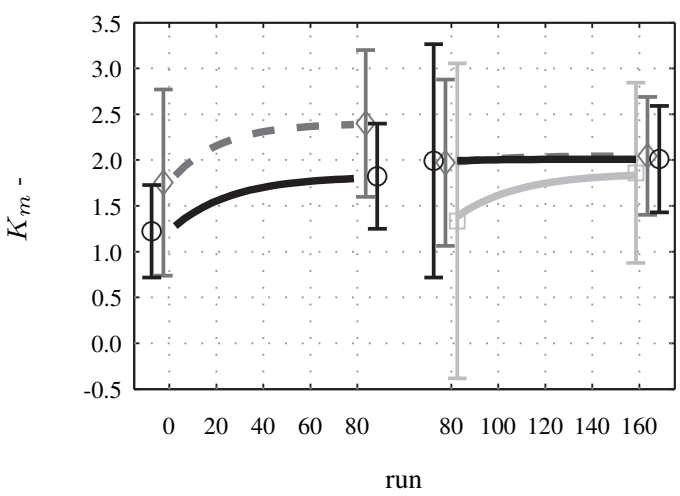

(f) Neuromuscular damping.

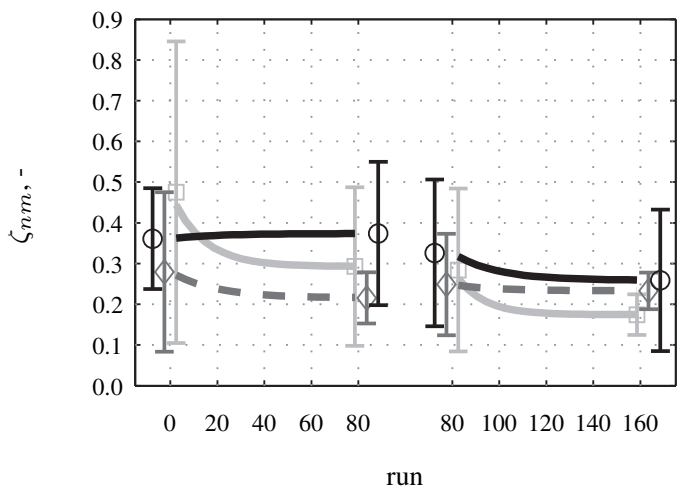

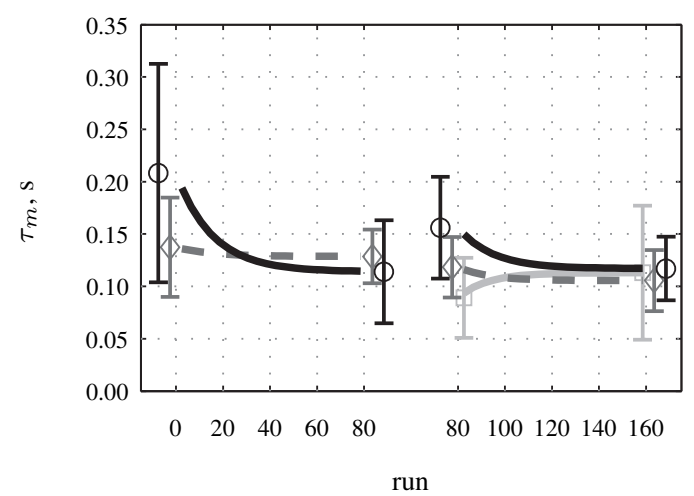

(g) Neuromuscular frequency.

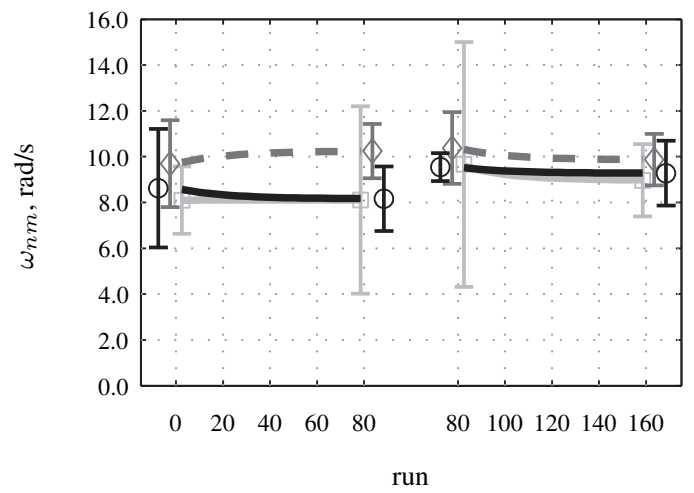

Figure 11. Pilot model parameters.

10 of 15 
(a) Pilot visual frequency response.
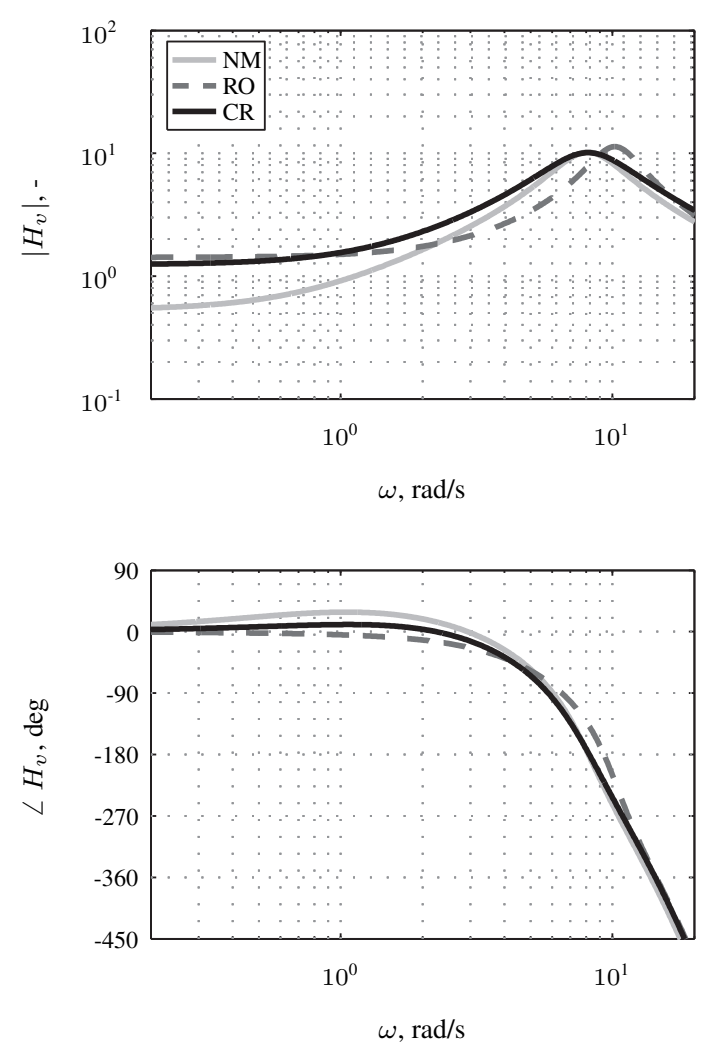

(b) Pilot motion frequency response.
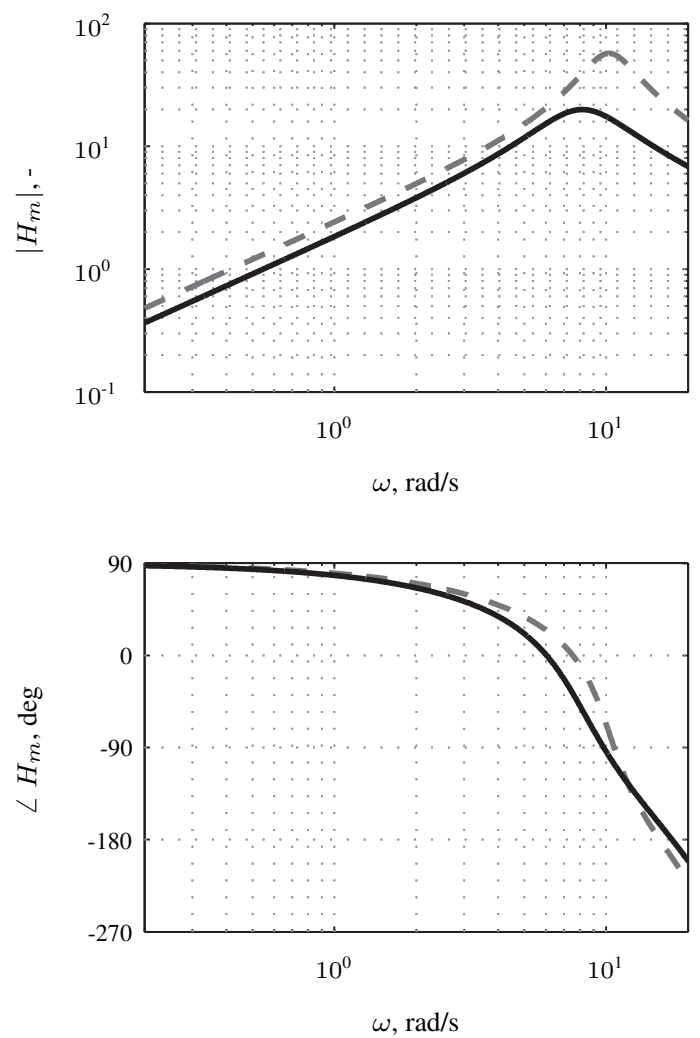

Figure 12. Mean pilot model frequency responses after training.

The evolution of the pilots' motion gain can be seen in Fig. 11d. Note that this parameter is estimated only when motion stimuli were present. During the training session, the motion gain shows an increasing trend. This suggests that pilots learn to act more confidently on the motion stimuli over the course of training. The roll-only group had the highest motion gain. In the transfer condition, no significant differences can be observed between the motion groups. However, pilots who trained without motion seem to have a lower motion gain at the beginning of the transfer session, which then increases during the session to the asymptotic level of the groups who trained with motion.

The remaining parameters presented in Fig. 11, all related to physical limitations of pilots, appear to be relatively unaffected by the motion used in training.

To clearly visualize the differences in obtained manual control skills after training with the three different motion configurations, Fig. 12 depicts the average frequency responses of the visual and motion channels for each training motion group at the end of the training session. These responses were calculated using the asymptotic values $N_{\infty}$ of each pilot model parameter in the training session with Eqs. 3 and 4.

The same observations can be made from Fig. 12 as from Fig. 11. Fig. 12a indicates that the no-motion group has the lowest gain at low frequencies, while the roll-only group has the highest gain. The no-motion group also starts to generate visual lead at lower frequencies, followed by the reduced-coordinated-roll and the roll-only groups. It can be observed that the gain of the motion response is higher for the roll-only group. Finally, it also seems that the neuromuscular peak frequency is slightly higher and the neuromuscular damping slightly lower for the roll-only motion group.

\section{IV.D. Crossover Frequencies and Phase Margins}

The open-loop frequency responses were calculated for both the disturbance and target forcing function inputs (Eqs. 5 and 6). Fig. 13 presents the disturbance and target crossover frequencies and phase margins. The difference between training motion conditions is more evident in the phase margin of the target open loop and in the crossover frequency 
(a) Target open loop.
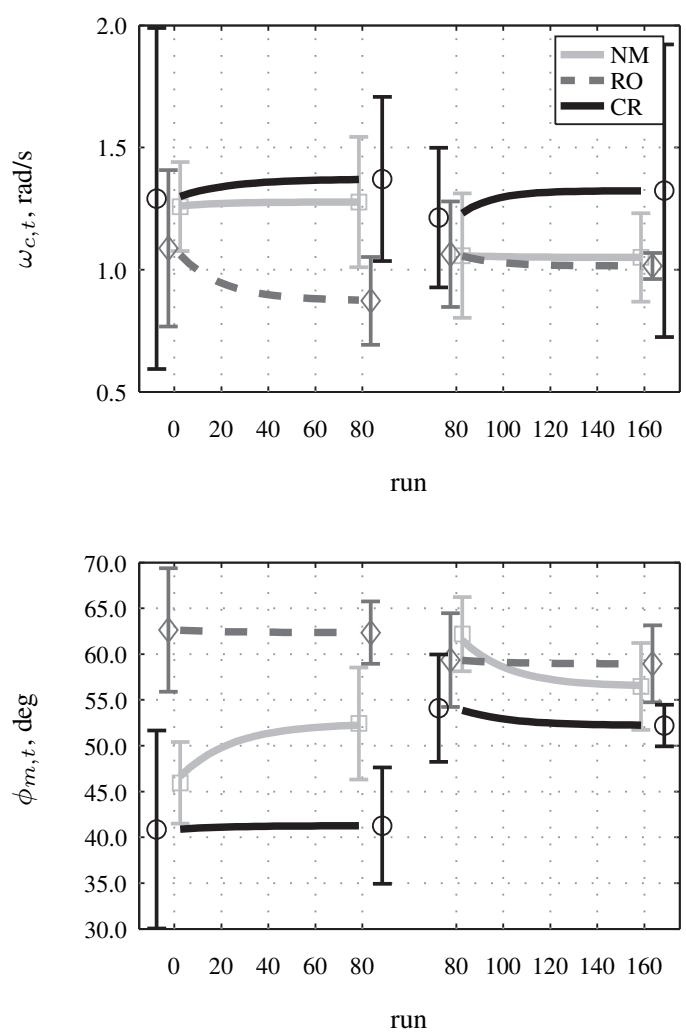

(b) Disturbance open loop.
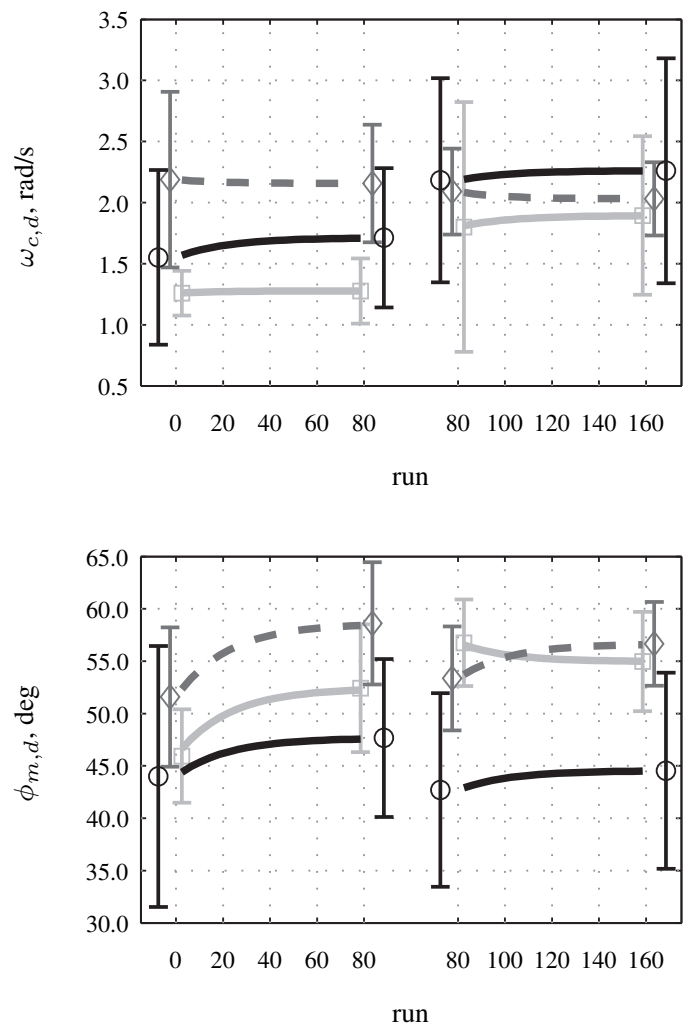

Figure 13. Open-loop characteristics.

of the disturbance open loop. These effects of motion on the disturbance crossover frequency and target phase margin have been observed in previous manual control studies. ${ }^{5}$

The disturbance crossover frequency is highest for the roll-only training condition and the lowest for the condition in which no motion was present. The roll-only training condition seems to yield the lowest value for the target crossover frequency at the end of training. The relative order of the target phase margin between motion groups in the training session is different from the order of the disturbance crossover frequency. The roll-only motion condition has the highest phase margin, while the coordinated roll condition shows the lowest phase margin. The phase margin of the no-motion training condition lies in-between. This relative order is also visible in the disturbance phase margin.

\section{Discussion}

This study investigated the effect of training with different motion configurations on the acquisition and transfer of manual control skills in a roll-tracking task. Pilots trained for half a day in one of three motion conditions - no motion, roll motion only, or reduced coordinated roll motion - and then transferred to full coordinated roll motion the next day. All other task variables, such as the controlled dynamics and forcing functions, were the same in both sessions.

The experiment had a between-subject design, with at least six pilots assigned to each motion group. However, one subject did not finish the experiment, two did not control the task according to standards and the data from two pilots did not allow for accurate pilot model parameter estimates. Eliminating these pilots resulted in having only four pilots for the no-motion and reduced-coordinated-roll groups. This, in combination with the between-subject design of the experiment, resulted in limited significant differences between motion groups in some of the dependent measures. In order to increase the significance of some of the observed differences, more pilots might be tested in the future.

As expected, training without motion results in the poorest performance in the training session, as indicated by the higher $R M S_{e}$ values. The tracking performance seems to be the best when pilots are trained with roll-only motion. Interestingly, tracking performance of the group who trained with reduced coordinated roll motion was very similar to the no-motion group. These differences between motion groups seem to be retained in the transfer session with full 
coordinated roll motion. The much better performance for the roll-only motion condition may partly be the result of the false tilt cues, which provided an increased sensation of motion. In addition, the roll motion gain for the roll-only motion condition was twice as large as the gain for the reduced-coordinated-roll motion condition. Similar observation can be made for the control activity. Without motion, pilots have a lower control activity in the training session, while pilots training with roll motion show higher levels of control activity. Similar trends between no-motion and motion conditions are observed in previous research. ${ }^{3}$ In the transfer condition, the pilots who trained with roll-only motion show a more consistent control intensity compared to the other training conditions. This might be attributed to the fact that the simulator roll gain is the same for training and transfer for this group.

The presence of large false cues in the roll-only condition has different effects on the investigated dependent measures. For instance, while performance was the highest when training with this motion condition, the highest VAF was achieved for the pilots training with reduced-coordinated-roll motion. One possible explanation is that, without turn coordination, the false tilt cue of the simulator induces biodynamic feedthrough, which reduces the linearity of pilot control inputs. Further analyzing the VAF, the starting values at the beginning of training seem to be very similar for all three motion groups. During training, however, pilots seem to be able to reduce their remnant, allowing for a larger portion of the control variance to be explained by the linear pilot model. This effect is reflected by the increasing trend of the VAF. This happens especially in the groups who trained with motion, suggesting that motion feedback allows for more linear behavior in this task. Although differences are not significant, the relative order of the VAF values at the end of the training session is maintained in the transfer session.

The effects of motion are reflected on the pilot model parameters when comparing the three different training motion conditions. Even though not necessarily apparent in Fig. 11, the trend of the parameter evolution is not always the same for every pilot. For instance, some pilots who started the experiment with a very high visual gain gradually decreased it over the course of training. The opposite trend was adopted for other pilots who started with a low visual gain in the same motion group. These large differences between pilots contributed to the large confidence intervals for the initial values of the exponential curves. At the end of training, however, pilots tend to approach the same level, resulting in smaller confidence intervals for the asymptotic values.

Having motion feedback increases the visual gain of pilots and replaces the need to obtain lead information from visual cues only. Pilots start generating visual lead at higher frequencies (lower lead time constant), since motion cues provide the required lead at lower frequencies. Pilot motion gains increase over the course of training, indicating that pilots gradually learn to use motion to their advantage. On the other hand, pilots who trained without motion show a larger change in motion gain in the transfer session than pilots who trained with motion. The roll-only configuration resulted in the highest visual gain, lowest lead time constant and the highest motion gain. This trend is also observed in previous research.

An extra remark can be made about the neuromuscular parameters observed at the end of the training session. Fig. 12 suggests that the roll-only motion condition results in a slightly higher neuromuscular frequency and lower neuromuscular damping at the end of training. This might also be attributed to possible biodynamic feedthrough, resulting from the false tilt cues.

The highest target forcing function phase margin is achieved with the roll-only training condition, followed by the no-motion condition. The reduced-coordinated-roll condition yields the lowest phase margin. Similar trends were found in a previous study, ${ }^{5}$ where the highest target phase margin was achieved with a roll gain of 1.0 and a break frequency of $0.0 \mathrm{rad} / \mathrm{s}$ and the lowest target phase margin for a roll gain of 0.5 and a break frequency of $0.5 \mathrm{rad} / \mathrm{s}$. These motion parameters are equivalent to the roll-only and reduced-coordinated-roll motion conditions of the current experiment. Event though the trends in the target phase margin are similar, differences were present in the controlled dynamics and the lateral motion cueing between the two experiments. This may suggest that roll motion fidelity has a bigger impact on the objective results than the fidelity of compensatory lateral motion.

The experiment contained three different training motion groups. In the roll-only-training-motion condition, the roll gain was 1.0 and no lateral motion was present to compensate for the false tilt cues. In the reduced-coordinatedroll-motion condition, no false tilt cues were present, but the roll gain was 0.5. The significant differences found between these two motion conditions might not only be the result of the false tilt cues, but could also just be caused by the difference in roll gain. To eliminate this confound in a future experiment, a roll-only condition should be added with a roll gain of 0.5 .

Pilots were asked to leave comments after the end of the training and transfer sessions. Notable comments were received by pilots who trained with the roll-only motion condition. They often mentioned that motion sensations were comparable to real flight even though large false cues were present. However, after performing the transfer session with the full coordinated roll motion, their opinion changed, stating that this motion configuration was even more realistic 
than the training. This might be caused by the lack of motion-base simulator experience of the general aviation pilots who participated in the experiment.

During the course of the experiment, a drop in performance often indicated the presence of fatigue. Improvements in performance were always observed after pilots took a short break. This indicates that fatigue affects the acquisition of manual control skills. To optimize the training of manual control skills, future work should also investigate optimal training procedures, such as the optimal length of training sessions and the number of training sessions.

\section{Conclusions}

An experiment was performed on the NASA Ames VMS to investigate the effect of different motion configurations on training and transfer of training of manual roll control skills. Of specific interest were the skills needed to control unstable roll dynamics of a mid-size transport aircraft close to the stall point. Three different motion configurations were used for training: no motion, uncoordinated roll motion, and reduced coordinated roll motion. All pilots transferred to full coordinated roll motion. The main difference between the motion configurations was the presence of large tilt miscues in the uncoordinated roll case. A novel multimodal pilot model identification technique was successfully applied to characterize how pilots' use of visual and motion cues changed over the course of training and after transfer. Pilots who trained with uncoordinated roll motion with false tilt cues had significantly higher performance during training and after transfer. Furthermore, pilot control behavior significantly changed during the two sessions, as indicated by increasing visual and motion gains, and decreasing lead time constants. Pilots training without motion showed higher learning rates after transfer to the full coordinated roll motion case. The results indicate that motion improves the training of manual roll control skills, even when false tilt cues are present.

\section{Acknowledgments}

The authors thank everybody at NASA Ames SimLabs who contributed to the experiment. We especially thank Emily Lewis, Deanna Doan, and Nick Riccobono for their valuable contributions.

\section{References}

${ }^{1}$ Zaal, P. M. T. and Sweet, B. T., “The Challenges of Measuring Transfer of Stall Recovery Training," Systems, Man and Cybernetics (SMC), 2014 IEEE International Conference on, Oct 2014, pp. 3138-3143.

${ }^{2}$ Stapleford, R. L., Peters, R. A., and Alex, F. R., "Experiments and a Model for Pilot Dynamics with Visual and Motion Inputs," Tech. Rep. NASA CR-1325, NASA, 1969.

${ }^{3}$ Zaal, P. M. T., Pool, D. M., de Bruin, J., Mulder, M., and van Paassen, M. M., "Use of Pitch and Heave Motion Cues in a Pitch Control Task," Journal of Guidance, Control, and Dynamics, Vol. 32, No. 2, March-April 2009, pp. 366-377.

${ }^{4}$ Schroeder, J. A. and Grant, P. R., "Pilot Behavioral Observations in Motion Flight Simulation," Proceedings of the AIAA Guidance, Navigation, and Control Conference and Exhibit, Toronto (ON), Canada, No. AIAA-2010-8353, 2-5 Aug. 2010.

${ }^{5}$ Pool, D. M., Zaal, P. M. T., Damveld, H. J., and van Paassen, M. M. M. M., "Evaluating Simulator Motion Fidelity using In-Flight and Simulator Measurements of Roll Tracking Behavior," Proceedings of the AIAA Modeling and Simulation Technologies Conference, Minneapolis (MN), No. AIAA-2012-4635, 13-16 Aug. 2012.

${ }^{6}$ Levison, W. H., Lancraft, R. E., and Junker, A. M., "Effects of Simulator Delays on Performance and Learning in a Roll-Axis Tracking Task," Fifteenth Annual Conference on Manual Control, Wright State University, Dayton (OH), 20-22 March 1979, pp. 168-186.

${ }^{7}$ Pool, D. M., Harder, G. A., Damveld, H. J., van Paassen, M. M., and Mulder, M., "Evaluating Simulator-Based Training of Skill-Based Control Behavior Using Multimodal Operator Models," Systems, Man and Cybernetics (SMC), 2014 IEEE International Conference on, Oct 2014, pp. 3132-3137.

${ }^{8}$ Jex, H. R., Jewell, W. F., and Magdaleno, R. E., "Effects of Various Lateral-Beam-Motion Washouts on Pilot Tracking and Opinion in the "Lamar" Simulator," Fifteenth Annual Conference on Manual Control, Wright State University, Dayton (OH), 20-22 March 1979, pp. $244-266$.

${ }^{9}$ Schroeder, J. A., "Helicopter Flight Simulation Motion Platform Requirements," Tech. Rep. NASA/TP-1999-208766, NASA, July 1999.

${ }^{10}$ McRuer, D. T., Graham, D., Krendel, E. S., and Reisener, W., "Human Pilot Dynamics in Compensatory Systems. Theory, Models and Experiments With Controlled Element and Forcing Function Variations," Tech. Rep. AFFDL-TR-65-15, Wright Patterson AFB (OH): Air Force Flight Dynamics Laboratory, 1965.

${ }^{11}$ Damveld, H. J., Beerens, G. C., van Paassen, M. M., and Mulder, M., "Design of Forcing Functions for the Identification of Human Control Behavior," Journal of Guidance, Control, and Dynamics, Vol. 33, No. 4, July - Aug. 2010, pp. 1064-1081.

${ }^{12}$ Danek, G. L., "Vertical Motion Simulator Familiarization Guide," NASA Technical Memorandum NASA TM-103923, NASA, 1993.

${ }^{13}$ Beard, S. D., Reardon, S. E., Tobias, E. L., and Aponso, B. L., "Simulation System Optimization for Rotorcraft Research on the Vertical Motion Simulator," Proceedings of the AIAA Modeling and Simulation Technologies Conference, Minneapolis (MN), No. AIAA-2012-4634, 1316 Aug. 2012.

${ }^{14}$ Pool, D. M., Zaal, P. M. T., van Paassen, M. M., and Mulder, M., "Effects of Heave Washout Settings in Aircraft Pitch Disturbance Rejection," Journal of Guidance, Control, and Dynamics, Vol. 33, No. 1, Jan.-Feb. 2010, pp. 29-41. 
${ }^{15}$ Zaal, P. M. T., Pool, D. M., Chu, Q. P., van Paassen, M. M., Mulder, M., and Mulder, J. A., "Modeling Human Multimodal Perception and Control Using Genetic Maximum Likelihood Estimation,” Journal of Guidance, Control, and Dynamics, Vol. 32, No. 4, July-Aug. 2009, pp. 1089-1099.

${ }^{16}$ Jex, H. R. and Magdaleno, R. E., "Roll Tracking Effects of G-vector Tilt and Various Types of Motion Washout," Fourteenth Annual Conference on Manual Control, University of Southern California, Los Angeles (CA), 25-27 April 1978, pp. 463-502.

\section{Appendix}

The table below shows a one-way analysis of variance (ANOVA) on the experimental dependent measures for each group of pilots. Before the analysis was conducted, checks for outliers, normal distribution, and homogeneity of variances were performed. Most of the data contains outliers, which were not removed due to the small pilot sample size; however, the majority of the data is normally distributed, as assessed by Shapiro-Wilk's test of normality $(p>0.05)$. There was homogeneity of variances for most of the data, as assessed by Levene's Test of Homogeneity, where $p>0.05$. In the future, more pilots could be tested to provide better consistency in the data.

Table 3. ANOVA results.

\begin{tabular}{|c|c|c|c|c|c|c|c|c|c|}
\hline & & \multicolumn{4}{|c|}{ Training } & \multicolumn{4}{|c|}{ Transfer } \\
\hline \multicolumn{2}{|c|}{ Measure } & $d f$ & $F$ & $p$ & Sig. & $d f$ & $F$ & $p$ & Sig. \\
\hline \multirow{3}{*}{$R M S_{e}$} & $N_{0}$ & $2.0,11.0$ & 0.864 & 0.448 & - & $2.0,11.0$ & 2.073 & 0.172 & - \\
\hline & $N_{\infty}$ & $2.0,11.0$ & 4.435 & 0.039 & $* *$ & $2.0,11.0$ & 0.396 & 0.683 & - \\
\hline & $\lambda$ & $2.0,11.0$ & 0.324 & 0.730 & - & $2.0,11.0$ & 0.429 & 0.662 & - \\
\hline \multirow{3}{*}{$R M S_{u}$} & $N_{0}$ & $2.0,11.0$ & 1.835 & 0.205 & - & $2.0,11.0$ & 0.790 & 0.478 & - \\
\hline & $N_{\infty}$ & $2.0,11.0$ & 4.706 & 0.033 & $* *$ & $2.0,11.0$ & 0.867 & 0.447 & - \\
\hline & $\lambda$ & $2.0,11.0$ & 0.209 & 0.815 & - & $2.0,11.0$ & 1.834 & 0.205 & - \\
\hline \multirow{3}{*}{$K_{v}$} & $N_{0}$ & $2.0,11.0$ & 3.527 & 0.066 & $*$ & $2.0,11.0$ & 1.565 & 0.252 & - \\
\hline & $N_{\infty}$ & $2.0,11.0$ & 12.485 & 0.001 & $* *$ & $2.0,11.0$ & 1.533 & 0.259 & - \\
\hline & $\lambda$ & $2.0,11.0$ & 0.568 & 0.582 & - & $2.0,11.0$ & 1.227 & 0.330 & - \\
\hline \multirow{3}{*}{$T_{L}$} & $N_{0}$ & $2.0,11.0$ & 18.808 & 0.000 & $* *$ & $2.0,11.0$ & 1.961 & 0.187 & - \\
\hline & $N_{\infty}$ & $2.0,11.0$ & 73.208 & 0.000 & $* *$ & $2.0,11.0$ & 0.287 & 0.756 & - \\
\hline & $\lambda$ & $2.0,11.0$ & 6.780 & 0.012 & $* *$ & $2.0,11.0$ & 13.327 & 0.001 & $* *$ \\
\hline \multirow{3}{*}{$\tau_{v}$} & $N_{0}$ & $2.0,11.0$ & 10.767 & 0.003 & $* *$ & $2.0,11.0$ & 2.060 & 0.174 & - \\
\hline & $N_{\infty}$ & $2.0,11.0$ & 1.501 & 0.265 & - & $2.0,11.0$ & 1.378 & 0.292 & - \\
\hline & $\lambda$ & $2.0,11.0$ & 15.250 & 0.001 & $* *$ & $2.0,11.0$ & 0.195 & 0.826 & - \\
\hline \multirow{3}{*}{$K_{m}$} & $N_{0}$ & $1.0,8.0$ & 1.085 & 0.328 & - & $1.0,8.0$ & 0.001 & 0.971 & - \\
\hline & $N_{\infty}$ & $1.0,8.0$ & 1.932 & 0.202 & - & $1.0,8.0$ & 0.011 & 0.919 & - \\
\hline & $\lambda$ & $1.0,8.0$ & 0.414 & 0.538 & - & $1.0,8.0$ & 0.025 & 0.878 & - \\
\hline \multirow{3}{*}{$\tau_{m}$} & $N_{0}$ & $1.0,8.0$ & 4.177 & 0.075 & * & $1.0,8.0$ & 4.132 & 0.077 & $*$ \\
\hline & $N_{\infty}$ & $1.0,8.0$ & 0.703 & 0.426 & - & $1.0,8.0$ & 0.511 & 0.495 & - \\
\hline & $\lambda$ & $1.0,8.0$ & 0.036 & 0.854 & - & $1.0,8.0$ & 0.298 & 0.600 & - \\
\hline \multirow{3}{*}{$\zeta_{n m}$} & $N_{0}$ & $2.0,11.0$ & 1.426 & 0.281 & - & $2.0,11.0$ & 0.509 & 0.615 & - \\
\hline & $N_{\infty}$ & $2.0,11.0$ & 3.345 & 0.073 & $*$ & $2.0,11.0$ & 1.741 & 0.220 & - \\
\hline & $\lambda$ & $2.0,11.0$ & 3.561 & 0.064 & $*$ & $2.0,11.0$ & 3.231 & 0.079 & $*$ \\
\hline \multirow{3}{*}{$\omega_{n m}$} & $N_{0}$ & $2.0,11.0$ & 1.354 & 0.298 & - & $2.0,11.0$ & 0.250 & 0.783 & - \\
\hline & $N_{\infty}$ & $2.0,11.0$ & 2.946 & 0.095 & $*$ & $2.0,11.0$ & 1.034 & 0.388 & - \\
\hline & $\lambda$ & $2.0,11.0$ & 1.022 & 0.392 & - & $2.0,11.0$ & 0.008 & 0.992 & - \\
\hline \multirow{3}{*}{$V A F$} & $N_{0}$ & $2.0,11.0$ & 0.054 & 0.948 & - & $2.0,11.0$ & 4.479 & 0.038 & $* *$ \\
\hline & $N_{\infty}$ & $2.0,11.0$ & 3.894 & 0.053 & $*$ & $2.0,11.0$ & 3.964 & 0.051 & $*$ \\
\hline & $\lambda$ & $2.0,11.0$ & 0.654 & 0.539 & - & $2.0,11.0$ & 0.201 & 0.821 & - \\
\hline \multirow{3}{*}{$\omega_{c, t}$} & $N_{0}$ & $2.0,11.0$ & 0.622 & 0.555 & - & $2.0,11.0$ & 0.944 & 0.418 & - \\
\hline & $N_{\infty}$ & $2.0,11.0$ & 10.794 & 0.003 & $* *$ & $2.0,11.0$ & 2.886 & 0.098 & $*$ \\
\hline & $\lambda$ & $2.0,11.0$ & 1.080 & 0.373 & - & $2.0,11.0$ & 1.106 & 0.365 & - \\
\hline \multirow{3}{*}{$\phi_{m, t}$} & $N_{0}$ & $2.0,11.0$ & 19.761 & 0.000 & $* *$ & $2.0,11.0$ & 4.185 & 0.000 & $* *$ \\
\hline & $N_{\infty}$ & $2.0,11.0$ & 40.901 & 0.000 & $* *$ & $2.0,11.0$ & 5.309 & 0.024 & $* *$ \\
\hline & $\lambda$ & $2.0,11.0$ & 0.768 & 0.487 & - & $2.0,11.0$ & 1.589 & 0.248 & - \\
\hline \multirow{3}{*}{$\omega_{c, d}$} & $N_{0}$ & $2.0,11.0$ & 4.195 & 0.044 & $* *$ & $2.0,11.0$ & 0.685 & 0.525 & - \\
\hline & $N_{\infty}$ & $2.0,11.0$ & 6.820 & 0.012 & $* *$ & $2.0,11.0$ & 0.795 & 0.476 & - \\
\hline & $\lambda$ & $2.0,11.0$ & 0.616 & 0.558 & - & $2.0,11.0$ & 0.851 & 0.453 & - \\
\hline \multirow{3}{*}{$\phi_{m, d}$} & $N_{0}$ & $2.0,11.0$ & 2.120 & 0.166 & - & $2.0,11.0$ & 10.410 & 0.003 & $* *$ \\
\hline & $N_{\infty}$ & $2.0,11.0$ & 6.141 & 0.016 & $* *$ & $2.0,11.0$ & 10.303 & 0.003 & ** \\
\hline & $\lambda$ & $2.0,11.0$ & 0.181 & 0.837 & - & $2.0,11.0$ & 3.376 & 0.072 & $*$ \\
\hline
\end{tabular}

